\title{
Serum Phthalate and Triclosan
} Levels Have Opposing Associations With Risk Factors for Gestational Diabetes Mellitus

OPEN ACCESS

Edited by:

Undurti Narasimha Das,

UND Life Sciences LLC,

United States

Reviewed by:

Akinobu Nakamura,

Hokkaido University, Japan

Federico Biscetti,

Università Cattolica del Sacro

Cuore, Italy

*Correspondence:

Carlo L. Acerini

cla22@cam.ac.uk

Specialty section:

This article was submitted

to Diabetes,

a section of the journal

Frontiers in Endocrinology

Received: 19 December 2017

Accepted: 28 February 2018

Published: 13 March 2018

Citation:

Fisher BG, Frederiksen $H$,

Andersson A-M, Juul $A$

Thankamony $A$, Ong $K K$, Dunger DB,

Hughes IA and Acerini CL (2018)

Serum Phthalate and Triclosan Levels

Have Opposing Associations

With Risk Factors for Gestational

Diabetes Mellitus.

Front. Endocrinol. 9:99.

doi: 10.3389/fendo.2018.00099

\author{
Benjamin G. Fisher ${ }^{1}$, Hanne Frederiksen ${ }^{2}$, Anna-Maria Andersson $^{2}$, Anders Juul'2, \\ Ajay Thankamony ${ }^{1}$, Ken K. Ong 1,3, David B. Dunger', leuan A. Hughes ${ }^{1}$ \\ and Carlo L. Acerini ${ }^{1 *}$
}

\begin{abstract}
${ }^{1}$ Department of Paediatrics, University of Cambridge, Cambridge, United Kingdom, ${ }^{2} E D M a R C$, Department of Growth and Reproduction, Rigshospitalet, University of Copenhagen, Copenhagen, Denmark, ${ }^{3} \mathrm{MRC}$ Epidemiology Unit, Institute of Metabolic Science, University of Cambridge, Cambridge, United Kingdom
\end{abstract}

Certain phthalates and bisphenol A (BPA) have been associated with insulin resistance and type 2 diabetes in non-pregnant adults, but studies of gestational diabetes mellitus (GDM) have reported conflicting results for phthalates and no associations with BPA. Our aim was to investigate the relationship between maternal serum levels of phthalate metabolites and phenols at 10-17 weeks of gestation and glucose homeostasis at 28 weeks of gestation. 232 women aged $\geq 16$ years without type 1 or 2 diabetes with singleton male pregnancies were recruited from a single UK maternity centre between 2001 and 2009 as part of a prospective observational study (Cambridge Baby Growth Study). Serum levels of 16 phthalate metabolites and 9 phenols (including BPA) were measured using liquid chromatography/tandem mass spectrometry. Oral glucose tolerance tests were performed at 28 weeks. 47/232 (20.3\%) women had GDM. First-trimester triclosan (TCS) was inversely associated with incident GDM (adjusted odds ratio per log increase in concentration $0.54,95 \%$ confidence interval 0.34-0.86, $p=0.010$ ). Amongst women without GDM, first-trimester mono-(2-ethylhexyl) phthalate and mono(carboxyisooctyl) phthalate levels were positively associated with 120-min plasma glucose (adjusted $\beta 0.268$ and $0.183, p=0.0002$ and 0.010 , respectively) in mid-pregnancy. No other monotonic associations were detected between phthalate or phenol levels and fasting or stimulated plasma glucose, $\beta$-cell function, insulin resistance, or 60-min disposition index. Our results support a glycaemia-raising effect of phthalates during pregnancy, consistent with findings in non-pregnant populations and suggest a possible protective effect of exposure to TCS against GDM.

\footnotetext{
Keywords: gestational diabetes, pregnancy, phthalates, bisphenol A, triclosan, endocrine disrupting chemicals, environmental chemicals

\begin{abstract}
Abbreviations: BP-3, benzophenone-3; BPA, bisphenol A; CBGS, Cambridge Baby Growth Study; DEHP, di-(2-ethylhexyl) phthalate; EDC, endocrine disrupting chemical; GDM, gestational diabetes mellitus; HOMA2, updated homeostasis model assessment; IGT, impaired glucose tolerance; IMD, Index of Multiple Deprivation 2007; LOD, limit of detection; MBzP, monobenzyl phthalate; MCiOP, mono(carboxyisooctyl) phthalate; MECPP, mono-(2-ethyl-5-carboxypentyl) phthalate; MEHP, mono-(2-ethylhexyl) phthalate; MEP, monoethyl phthalate; MiBP, monoisobutyl phthalate; MnBP, mono-n-butyl phthalate; OGTT, oral glucose tolerance test; T2DM, type 2 diabetes mellitus.
\end{abstract}




\section{INTRODUCTION}

Late pregnancy is characterised by peripheral insulin resistance with a compensatory increase in insulin secretion and $\beta$-cell mass. Inadequacy of this response leads to gestational diabetes mellitus (GDM), which is associated with both perinatal and long-term adverse outcomes (1). The incidence of GDM in developed countries has increased significantly in recent years, a trend that cannot be fully explained by rising levels of maternal obesity (2). There is increasing interest in a possible role for endocrine disrupting chemicals (EDCs), exogenous substances that interfere with the endocrine system and cause adverse health effects. Two groups of EDCs that have been well studied in the context of glycaemia are phthalic acid esters (phthalates) and bisphenol A (BPA, 4,4'-dihydroxy-2,2-diphenylpropane).

Phthalates are ubiquitous industrial chemicals, several metabolites of which are detectable in the urine of $>75 \%$ of the general population (3). In vitro, phthalates activate peroxisome proliferator-activated receptors, which regulate cellular lipid and glucose metabolism (4), and reduce insulin receptor concentration (5). In rodents, phthalates impair insulin secretion and glucose tolerance (6). Epidemiological studies in non-pregnant adults have demonstrated associations between phthalates and raised fasting blood glucose, insulin resistance, reduced insulin secretion, and type 2 diabetes mellitus (T2DM) (7-9). To date, only three epidemiological studies have assessed the associations between phthalates and gestational glycaemia. In a cohort of 72 women, first-trimester urinary concentrations of monoisobutyl phthalate (MiBP) and monobenzyl phthalate $(\mathrm{MBzP})$ were inversely associated with 60-min blood glucose levels measured 3-25 weeks later (10). In a larger study $(n=350)$, second-trimester urinary monoethyl phthalate (MEP) was positively associated with impaired glucose tolerance (IGT), whereas di-(2-ethylhexyl) phthalate (DEHP) metabolites were associated with lower odds of IGT (11). The largest study $(n=1,274)$ observed no significant associations between first-trimester urinary phthalate metabolites and incident IGT/GDM (12).

Bisphenol A is another commonly used industrial chemical, detectable in the urine of $93 \%$ of the US population (13). It exerts oestrogenic and various other effects (7). In rodents, BPA causes disrupted insulin signalling, insulin resistance, and IGT (14). Epidemiological studies in non-pregnant adults have generated conflicting results for associations between BPA and insulin resistance and T2DM $(9,15-17)$. In the three studies of pregnant women to date, only one prospective cohort study $(n=245)$ reported a positive association between second-trimester (but not first-trimester) urinary BPA levels and stimulated blood glucose levels (but not incident GDM) at 24-28 weeks of gestation (18). In a larger study $(n=1,274)$, first-trimester urinary BPA was not significantly associated with incident IGT/GDM (12), and in a small case-control study $(n=94)$, second-trimester urinary BPA was not associated with GDM or fasting blood glucose levels amongst controls (19).

Other phenol compounds [4-tert-octyl phenol, benzophenone-3 (BP-3), and triclosan (TCS)] have not been associated with T2DM or fasting serum glucose in humans (20).
Our objective was to investigate the prospective relationships between maternal serum levels of phthalate metabolites, BPA, and other phenols at 10-17 weeks of gestation and glucose homeostasis at 28 weeks of gestation in a UK setting.

\section{MATERIALS AND METHODS}

\section{Study Design}

Pregnant women aged $\geq 16$ years $(n=2,229)$ were recruited to the Cambridge Baby Growth Study (CBGS), a large prospective cohort study, from routine antenatal clinics at 10-17 weeks of gestation at the Rosie Maternity Unit, Cambridge, between April 2001 and March 2009. Characteristics of CBGS participants have previously been described (21). Written informed consent was obtained from all women.

A subcohort of 330 mothers of 334 male infants was selected for a nested case-control study of EDCs and cryptorchidism (30 infants with congenital cryptorchidism, 21 infants with postnatally acquired cryptorchidism, 285 controls). Of these, women were retrospectively included in the present study if they had a singleton pregnancy, no pre-existing diagnosis of type 1 or 2 diabetes mellitus or GDM, and undertook an oral glucose tolerance test (OGTT) at 28 weeks of gestation. This subset of 232 women was representative of the whole cohort with regard to age, ethnicity, pre-pregnancy body mass index (BMI), and deprivation index (all $p>0.2)$.

Non-fasting maternal serum samples were collected into plastic-free glass tubes at enrolment for measurement of 16 phthalate metabolites and 9 phenols (Table 1). Serum samples were stored at $-70^{\circ} \mathrm{C}$. OGTTs were performed to diagnose GDM and assess $\beta$-cell function and insulin resistance.

\section{Maternal Demographic Characteristics}

A questionnaire administered at $\sim 36$ weeks of gestation collected demographic information, including history of diabetes mellitus, ethnicity, smoking, and parity. The Index of Multiple Deprivation 2007 (IMD), which measures various economic, social, and housing parameters, was derived from residential postcodes (22). The sample mean IMD value (9.37) was imputed for those cases where it was missing $(n=54)$ as these women did not differ from others with regard to age, pre-pregnancy BMI, ethnicity, smoking status, parity, or prevalence of GDM (all $p>0.3$ ).

\section{Oral Glucose Tolerance Testing}

At 28 weeks of gestation, a 75-g OGTT was performed following an overnight fast. Venous whole blood glucose levels were measured at 0 and $60 \mathrm{~min}$, and capillary blood glucose levels were measured at 0, 60, and $120 \mathrm{~min}$. From May 2007 onwards, venous whole blood glucose levels were also measured at $120 \mathrm{~min}$ (in 56/232 women, 24.1\%). Women completing OGTTs before May 2007 did not differ from those completing OGTTs during/after May 2007 with regard to age, BMI, IMD, ethnicity, smoking status, parity, or prevalence of GDM (all $p>0.1$ ). Insulin and C-peptide levels were measured at 0 and $60 \mathrm{~min}$ in 158/232 (68.1\%) women. Women with missing insulin and C-peptide levels did not differ 
TABLE 1 | Medians, quartiles, and ranges of serum concentrations of the investigated phthalate metabolites and phenols in the study population $(n=232$ for phthalates, $n=228$ for phenols).

\begin{tabular}{|c|c|c|c|c|c|c|c|}
\hline \multirow[t]{2}{*}{ Chemical concentration $(\mu \mathrm{g} / \mathrm{l})^{\mathrm{a}}$} & \multirow{2}{*}{$\begin{array}{l}\text { Limit of detection } \\
\text { (LOD) }\end{array}$} & \multirow{2}{*}{$\begin{array}{l}\text { Detection } \\
\text { rate (\%) }\end{array}$} & \multirow[t]{2}{*}{ Minimum } & \multicolumn{3}{|c|}{ Percentiles } & \multirow[t]{2}{*}{ Maximum } \\
\hline & & & & 25th & 50th & 75th & \\
\hline \multicolumn{8}{|l|}{ Phthalate metabolites [parent compound] } \\
\hline Monoethyl phthalate (MEP) [DEP] $]^{b}$ & 0.50 & 90.5 & $<$ LOD & 0.79 & 1.56 & 4.26 & 172.05 \\
\hline Monoisobutyl phthalate (MiBP) [DiBP] ${ }^{\mathrm{b}}$ & 0.77 & 97.8 & $<$ LOD & 1.97 & 3.78 & 6.60 & 29.34 \\
\hline Mono-n-butyl phthalate (MnBP) [DnBP] $]^{b}$ & 0.82 & 71.6 & & $<$ LOD & 1.34 & 2.31 & 9.41 \\
\hline Mono-pentyl phthalate (MPP) [DPP] ${ }^{\mathrm{b}}$ & 0.20 & 0.4 & & & & $<L O D$ & 0.24 \\
\hline Monobenzyl phthalate (MBzP) [BBzP] ${ }^{b}$ & 0.40 & 6.9 & & & & $<L O D$ & 1.08 \\
\hline Mono-(2-ethylhexyl) phthalate (MEHP) [DEHP] ${ }^{\mathrm{b}}$ & 0.60 & 63.8 & & $<\mathrm{LOD}$ & 1.14 & 58.53 & 421.93 \\
\hline Mono-(2-ethyl-5-hydroxyhexyl) phthalate (MEHHP) [DEHP] & 0.56 & 6.9 & & & & $<L O D$ & 4.45 \\
\hline Mono-(2-ethyl-5-oxohexyl) phthalate (MEOHP) [DEHP] & 0.35 & 2.3 & & & & $<L O D$ & 1.94 \\
\hline Mono-(2-ethyl-5-carboxypentyl) phthalate (MECPP) [DEHP] & 0.28 & 79.3 & $<$ LOD & 0.30 & 0.52 & 0.95 & 14.39 \\
\hline Mono-n-octyl phthalate (MOP) [DnOP] $]^{\mathrm{b}}$ & 0.20 & 0.4 & & & & $<L O D$ & 0.26 \\
\hline Mono-(3-carboxypropyl) phthalate (MCPP) [DnOP] & 0.36 & 0.4 & & & & $<L O D$ & 0.44 \\
\hline Mono-isononyl phthalate (MiNP) [DiNP] ${ }^{\mathrm{b}}$ & 0.20 & 19.0 & & & & $<$ LOD & 3.24 \\
\hline Mono(hydroxyisononyl) phthalate (MHiNP) [DiNP] & 0.38 & 0.0 & & & & & $<$ LOD \\
\hline Mono-oxoisononyl phthalate (MOiNP) [DiNP] & 0.10 & 3.4 & & & & $<L O D$ & 0.22 \\
\hline Mono(carboxyisooctyl) phthalate (MCiOP) [DiNP] & 0.10 & 75.4 & $<$ LOD & 0.10 & 0.18 & 0.37 & 13.76 \\
\hline Mono-isodecyl phthalate (MiDP) [DiDP] ${ }^{\mathrm{b}}$ & 0.20 & 3.4 & & & & $<L O D$ & 0.43 \\
\hline \multicolumn{8}{|l|}{ Phenols } \\
\hline Bisphenol A (BPA) & 0.18 & 89.9 & $<L O D$ & 1.02 & 1.76 & 2.96 & 8.46 \\
\hline Triclosan (TCS) & 0.22 & 69.3 & & $<$ LOD & 0.93 & 8.29 & 178.75 \\
\hline Triclocarban (TCC) & 0.27 & 26.3 & & & $<L O D$ & 0.36 & 18.85 \\
\hline Benzophenone-3 (BP-3) & 0.12 & 70.2 & & $<$ LOD & 0.34 & 0.79 & 67.11 \\
\hline 2,4-dichlorophenol (2,4-DCP) & 0.18 & 11.4 & & & & $<L O D$ & 0.69 \\
\hline 2,5-dichlorophenol (2,5-DCP) & 0.11 & 4.5 & & & & $<L O D$ & 3.88 \\
\hline 2,4,5-trichlorophenol (2,4,5-TCP) & 0.29 & 0.4 & & & & $<$ LOD & 1.26 \\
\hline 2-phenylphenol (2-PP) & 0.13 & 41.2 & & & $<L O D$ & 0.23 & 0.74 \\
\hline 4-phenylphenol (4-PP) & 0.12 & 5.7 & & & & $<L O D$ & 0.37 \\
\hline
\end{tabular}

${ }^{a}<L O D$ : below the $L O D$.

${ }^{b}$ Semiquantitative data (see Materials and Methods).

from others with regard to age, BMI, IMD, ethnicity, smoking status, parity, or prevalence of GDM (all $p>0.1$ ). Glycated haemoglobin levels were not measured.

\section{Assays}

We measured phthalate metabolites rather than their diester parent compounds, as the latter are metabolised rapidly (23), and exposure misclassification could potentially occur from contamination of the samples with phthalate diesters during venesection or laboratory handling. Serum samples were analysed for the total content of 16 phthalate metabolites (from 9 parent phthalate diesters) and 9 phenols (Table 1) by isotope-diluted liquid chromatography coupled to tandem mass spectrometry. The method and determination of the limit of detection (LOD) was as previously described $(24,25)$ (Supplementary Methods in Supplementary Material). Measured levels of the phthalate monoesters were considered to be semiquantitative, as hydrolysis of potentially contaminating diester phthalates to monoester phthalates [e.g., DEHP to mono-(2-ethylhexyl) phthalate (MEHP)] by enzymes present in blood may have occurred after the samples were drawn. However, the large interindividual variation observed for these metabolites suggests that a systematic contamination of the samples with one or more of the phthalate diesters is unlikely. The measured levels for downstream phthalate metabolites [mono-(2-ethyl-5-hydroxyhexyl) phthalate (MEHHP), mono-(2-ethyl-5-oxohexyl) phthalate, mono-(2-ethyl-5-carboxypentyl) phthalate (MECPP), mono(3-carboxypropyl) phthalate, mono(hydroxyisononyl) phthalate, mono-oxoisononyl phthalate, mono(carboxyisooctyl) phthalate $(\mathrm{MCiOP})]$ were quantitative, as conversion to these metabolites can occur only in vivo.

Whole blood glucose levels were measured using a standard glucose oxidase-based assay. Capillary blood glucose measurements were made using an Abbott Freestyle Mini (Abbott Diagnostics, Maidenhead, UK). Fasting and stimulated insulin and C-peptide levels were measured by enzyme-linked immunosorbent assay using commercial kits (DSL, London, UK).

\section{Calculations}

Analyses of associations with parameters of blood glucose homeostasis included only those chemicals measured above the LOD in $>60 \%$ of samples. Concentrations below the LOD were assigned a value equal to $\mathrm{LOD} / 2$ if the data were highly skewed or LOD $/ \sqrt{ } 2$ if not (26). These substituted data were then used for all subsequent analyses. Concentrations were log-transformed to correct for non-normal distributions with positive skew. For categorical analyses, subjects were categorised into quartiles using the 25 th, 50th, and 75 th percentiles of the subcohort $(n=232)$, with the first quartile used as the referent category.

For the purposes of diagnosing GDM and estimating $\beta$-cell function and insulin resistance, venous plasma glucose levels were estimated by multiplying venous whole blood glucose 
levels, where available, by 1.08 , assuming a haematocrit of $31.5 \%(27,28)$. For the 176 women who completed an OGTT prior to May 2007 and, therefore, did not have a 120-min venous whole blood glucose level measured, 120-min venous plasma glucose level was estimated using the equation venous plasma glucose $=1.035 \times$ capillary blood glucose -0.891 , which was derived from a linear regression model assessing the ability of 60 -min capillary blood glucose to predict 60 -min venous plasma glucose using data from the entire CBGS cohort $\left[R^{2}=0.790, F(1,768)=2,889.3, p=2 \times 10^{-262}\right]$. A linear regression model assessing the ability of fasting capillary blood glucose to predict fasting venous plasma glucose gave a different result: venous plasma glucose $=0.826 \times$ capillary blood glucose $+0.996\left[R^{2}=0.511, F(1,759)=792.6, p=6 \times 10^{-120}\right]$; however, we justified using the first equation to estimate the missing 120-min venous plasma glucose levels as across the entire CBGS cohort the mean 60 -min venous plasma glucose (7.4 $\mathrm{mmol} / \mathrm{l})$ was closer to the mean 120 -min venous plasma glucose $(6.9 \mathrm{mmol} / \mathrm{l})$ than the mean fasting venous plasma glucose $(4.7 \mathrm{mmol} / \mathrm{l})$ The updated homeostasis model assessment (HOMA2) was used to estimate steady-state $\beta$-cell function (HOMA2-B, using fasting C-peptide and plasma glucose levels) and insulin resistance (HOMA2-IR, using fasting insulin and plasma glucose levels) (29); software for calculating these values is available online (https://www.dtu.ox.ac.uk/ homacalculator/). To estimate insulin secretion independent of insulin sensitivity, we calculated the disposition index as the 60 -min insulinogenic index [(insulin 60 - insulin 0 )/(venous glucose 60 - venous glucose 0)] divided by HOMA2-IR (30). HOMA2-B, HOMA2-IR, and disposition index were logtransformed to normalise distributions.

\section{Diagnosis of GDM}

For primary analyses, women were diagnosed with GDM if they met one or more of the following criteria: fasting plasma glucose $\geq 5.1 \mathrm{mmol} / \mathrm{l}, 60$ - $\mathrm{min}$ plasma glucose $\geq 10.0 \mathrm{mmol} / 1$, or 120 - $\mathrm{min}$ plasma glucose $\geq 8.5 \mathrm{mmol} / \mathrm{l}(31,32)$. For sensitivity analyses, the following criteria were used: fasting plasma glucose $\geq 7.0 \mathrm{mmol} / 1$ or 120 -min plasma glucose $\geq 7.8 \mathrm{mmol} / \mathrm{l}$ (33).

\section{Neonatal Examinations}

Newborns were examined by research nurses, as far as possible in the first 2 weeks of life, either in hospital or at home visits. They were then re-examined during clinic visits at 3,12 , and 24 months of age. Birth weight as measured at delivery by midwives was taken from health records. Cryptorchidism was defined as one or more testes not present in the inferior half of the scrotum for at least a few moments after manipulation.

\section{Ethical Approval}

The study was approved by Cambridge Local Research Ethics Committee and adhered to the Declaration of Helsinki.

\section{Statistical Analyses}

Distribution percentiles of phthalate and phenol concentrations were calculated for women with and without GDM, and differences in patient characteristics were assessed using the $t$-test and
Mann-Whitney $U$-test for continuous variables, and chi-square test for categorical variables.

Logistic regression was used to model the odds of GDM in relation to serum phthalate/phenol concentrations, adjusting for covariates of interest. Continuous relationships between phthalate/phenol levels and HOMA2-B, HOMA2-IR, fasting and 120 -min plasma glucose, and disposition index were individually assessed using multiple linear regression, adjusting for covariates of interest. We restricted these latter analyses to women who did not have GDM, because in women with GDM glucose toxicity per se may cause insulin resistance and $\beta$-cell dysfunction (8). Outliers (data points with standardised residuals $>3$ SDs) were removed in all regressions (typically $0-2$ cases per regression). Tests of heterogeneity $\left(p_{\text {het }}\right)$ and linear trend $\left(p_{\text {trend }}\right)$ in outcome value across serum phthalate/phenol concentration quartiles were performed. Because numerous chemicals were significantly correlated and several have been previously associated with dysglycaemia (7-11, 15-17), regression analyses were conducted separately for each chemical, and we did not correct for multiple testing.

The following potential confounders were chosen a priori as covariates in the multivariable analyses because of their known biological relevance to the outcomes of interest (12): age, prepregnancy BMI (log-transformed to normalise the distribution), IMD (log-transformed), and parity (nulliparous vs. para 1 vs. para 2 or greater). We did not use ethnicity or smoking status in our regressions, because very few women were of non-white ethnicity $(4 / 151,2.6 \%)$ or were smokers $(5 / 232,2.2 \%)$, and data on ethnicity were missing for $81 / 232$ (34.9\%) women.

In view of the nature of our subcohort (case-control study of cryptorchidism) and a possible association between GDM and congenital cryptorchidism (34), we additionally used logistic regression to model the odds of GDM in relation to cryptorchidism at birth, and multiple linear regression to assess the relationship between cryptorchidism and HOMA2-B, HOMA2-IR, fasting/ stimulated plasma glucose, and disposition index in women who did not have GDM, adjusting all analyses for the above covariates as well as prematurity (gestation $<37$ vs. $\geq 37$ weeks) and small for gestational age (birth weight SD score $<-1.5$ vs. $\geq-1.5$ ), which are both risk factors for cryptorchidism (34). Finally, we used logistic regression to remodel the odds of GDM in relation to serum phthalate/phenol concentrations after excluding mothers of sons with congenital cryptorchidism.

All $p$-values were two-sided and $p<0.05$ was considered statistically significant. Data were analysed using SPSS, version 22.0 (IBM Corporation, New York, NY, USA).

\section{RESULTS}

232 women participated in the study, of whom 47 (20.3\%) met one or more criteria for GDM (Table 2). Of these, $34(72.3 \%)$ had an abnormal fasting plasma glucose, 18 (38.3\%) had an abnormal 60-min glucose, and 14 (29.8\%) had an abnormal 120-min glucose.

Six phthalate metabolites [MEP, MiBP, mono-n-butyl phthalate (MnBP), MEHP, MECPP, MCiOP] and three phenols (BPA, triclosan, BP-3) were detectable in $>60 \%$ of samples (Table 1; 
TABLE 2 | Characteristics of the study population.

\begin{tabular}{|c|c|c|c|}
\hline Characteristics $^{\mathrm{a}}$ & $\begin{array}{l}\text { Women with } \\
\text { gestational } \\
\text { diabetes mellitus } \\
\text { (GDM) }(n=47)\end{array}$ & $\begin{array}{l}\text { Women } \\
\text { without } \\
\text { GDM } \\
(n=185)\end{array}$ & $\begin{array}{l}p \text {-Value } \\
\text { for GDM } \\
\text { vs. no } \\
\text { GDM }^{\mathrm{b}}\end{array}$ \\
\hline Age (years) & $33.1 \pm 4.4$ & $33.7 \pm 3.8$ & 0.30 \\
\hline $\begin{array}{l}\text { Pre-pregnancy body } \\
\text { mass index }\left(\mathrm{kg} / \mathrm{m}^{2}\right)\end{array}$ & $25.4 \pm 5.1$ & $23.7 \pm 3.7$ & 0.051 \\
\hline \multicolumn{4}{|l|}{ Ethnicity $^{c}$} \\
\hline White & 28 (100\%) & 119 (96.7\%) & 0.75 \\
\hline Other & 0 (0\%) & 4 (3.3\%) & \\
\hline \multicolumn{4}{|l|}{ Current smoker } \\
\hline No & 46 (97.9\%) & $181(97.8 \%)$ & 0.99 \\
\hline Yes & $1(2.1 \%)$ & $4(2.2 \%)$ & \\
\hline \multicolumn{4}{|l|}{ Parity } \\
\hline 0 & 21 (44.7\%) & 87 (47.3\%) & 0.91 \\
\hline 1 & 20 (42.6\%) & $72(39.1 \%)$ & \\
\hline$\geq 2$ & $6(12.8 \%)$ & $25(13.6 \%)$ & \\
\hline $\begin{array}{l}\text { Index of multiple } \\
\text { deprivation }(U)\end{array}$ & $9.43 \pm 3.48$ & $9.35 \pm 4.27$ & 0.90 \\
\hline $\begin{array}{l}\text { Fasting plasma } \\
\text { glucose (mmol/l) }\end{array}$ & $5.5 \pm 0.9$ & $4.5 \pm 0.3$ & $4 \times 10^{-9 *}$ \\
\hline $\begin{array}{l}\text { 60-min plasma } \\
\text { glucose }(\mathrm{mmol} / \mathrm{l})\end{array}$ & $9.5 \pm 2.0$ & $7.1 \pm 1.5$ & $1 \times 10^{-10 *}$ \\
\hline $\begin{array}{l}\text { 120-min plasma } \\
\text { glucose (mmol/l) }\end{array}$ & $7.6 \pm 1.7$ & $5.9 \pm 1.0$ & $3 \times 10^{-8 *}$ \\
\hline HOMA2-B (\%) & 113.7 (94.7-125.9) & $120.0(106.0-146.1)$ & 0.07 \\
\hline HOMA2-IR (U) & 1.35 (0.96-1.68) & $0.82(0.67-1.08)$ & $2 \times 10^{-6 *}$ \\
\hline $\begin{array}{l}\text { Disposition index } \\
(\mathrm{mmol} / \mathrm{pmol} . U)\end{array}$ & 119.7 (59.6-160.2) & 152.7 (112.0-284.9) & $0.001^{*}$ \\
\hline \multicolumn{4}{|c|}{$\begin{array}{l}\text { Infant with congenital } \\
\text { cryptorchidism }\end{array}$} \\
\hline No & $43(91.5 \%)$ & 173 (93.5\%) & 0.87 \\
\hline Yes & $4(8.5 \%)$ & 12 (6.5\%) & \\
\hline
\end{tabular}

aMean \pm SD or median (IQR) for continuous variables, $n$ (\%) for categorical variables. ${ }^{b}$ Mann-Whitney U-test for HOMA2-B, HOMA2-IR, and disposition index; $t$-test for other continuous variables; chi-square test for categorical variables (with continuity correction for $2 \times 2$ tables).

${ }^{\circ}$ Data on ethnicity are missing for $n=81$ women. ${ }^{*} p<0.05$.

Table S1 in Supplementary Material). Some phthalate metabolites were strongly or moderately intercorrelated (MnBP and MiBP, rho $=0.838, p=2 \times 10^{-62}$; MECPP and MCiOP, rho $=0.357$, $\left.p=2 \times 10^{-8}\right)$, and several of the other chemicals were weakly correlated (Table S1 in Supplementary Material).

In continuous models, of these nine chemicals, only triclosan was associated with incident GDM [adjusted OR (aOR) per log increase in concentration $0.54,95 \%$ CI $0.34-0.86, p=0.010$ ] (Table 3). We conducted a categorical analysis to look for threshold and non-linear effects (Table 4). In accordance with the continuous analysis, this demonstrated lower odds of GDM in the second ( $\mathrm{aOR} 0.25,95 \%$ CI $0.07-0.86, p=0.028$ ), third (aOR 0.12, 95\% CI 0.03-0.55, $p=0.007$ ), and fourth (aOR 0.35 , $95 \%$ CI $0.12-0.98, p=0.046)$ quartiles for triclosan, compared to the first quartile $\left(p_{\text {het }}=0.009, p_{\text {trend }}=0.022\right.$ ). Inspection of the data did not reveal evidence for a threshold or non-linear relationship. Conversely, women with MiBP levels in the second (aOR 5.69, 95\% CI 1.56-20.73, $p=0.008$ ) and fourth (aOR 4.89, 95\% CI 1.32-18.14, $p=0.018$ ) quartiles, but not in the third quartile, had higher odds of GDM $\left(p_{\text {het }}=0.001\right.$, $\left.p_{\text {trend }}=0.41\right)$.
TABLE 3 | Adjusted odd ratios and 95\% Cls for incident gestational diabetes mellitus in relation to log-transformed serum chemical concentrations.

\begin{tabular}{lcc} 
Log-transformed serum chemical & \multicolumn{2}{c}{ Adjusted model $^{\mathrm{a}}$} \\
\cline { 2 - 3 } concentration & OR (95\% Cl) & p-Value \\
\hline Monoethyl phthalate & $0.81(0.39-1.70)$ & 0.58 \\
Monoisobutyl phthalate & $1.48(0.51-4.34)$ & 0.47 \\
Mono-n-butyl phthalate & $1.55(0.45-5.33)$ & 0.49 \\
Mono-(2-ethylhexyl) phthalate & $0.93(0.66-1.31)$ & 0.67 \\
Mono-(2-ethyl-5-carboxypentyl) phthalate & $0.75(0.27-2.06)$ & 0.57 \\
Mono(carboxyisooctyl) phthalate & $1.12(0.47-2.66)$ & 0.81 \\
Bisphenol A & $1.16(0.48-2.78)$ & 0.74 \\
Triclosan & $0.54(0.34-0.86)$ & $0.010^{*}$ \\
Benzophenone-3 & $0.80(0.44-1.44)$ & 0.45
\end{tabular}

${ }^{a}$ Adjusted for age, pre-pregnancy body mass index (log-transformed), IMD (log-transformed), and parity; $n=197$ for phthalates, $n=193$ for phenols. ${ }^{*} p<0.05$.

To explore the robustness of our results, and in view of the high incidence of GDM in our sample compared to previous studies (2), we repeated our analyses for MiBP and triclosan using the more stringent 1999 World Health Organization (WHO) criteria for GDM, i.e., fasting plasma glucose $\geq 7.0 \mathrm{mmol} / \mathrm{l}$ or 120 - min plasma glucose $\geq 7.8 \mathrm{mmol} / \mathrm{l}$ (33). Using these criteria, the incidence of GDM was 27/232 (11.6\%). The inverse association between incident GDM and triclosan persisted in both the continuous analysis (aOR 0.25 , 95\% CI 0.09-0.65, $p=0.005$ ) and the categorical analysis $\left(p_{\text {het }}=0.28, p_{\text {trend }}=0.006\right)$. However, there was no significant association between MiBP levels and incident GDM (data not shown).

Among women without GDM at 28 weeks of gestation $(n=185)$, MEHP was positively associated with 120 -min plasma glucose (adjusted $\beta 0.268, p=0.0002$ ), explaining $6.9 \%$ of the variation in 120-min glucose (Table S2 in Supplementary Material). MCiOP was also positively associated with 120 -min glucose (adjusted $\beta 0.183, p=0.010$ ), explaining $3.4 \%$ of the variation in 120-min glucose. Other phthalate metabolites (including MiBP) and all phenols were not significantly associated with fasting/ stimulated glucose levels, HOMA2-B, HOMA2-IR, or disposition index (Table S2 in Supplementary Material).

Experimental studies suggest that MEHP, the primary metabolite of DEHP, may have greater endocrine disrupting activity that is secondary metabolites, such as MECPP (35). We, therefore, tested the associations between the molar ratio of MEHP to MECPP (MEHP/MECPP ratio), as an inverse marker of detoxification capability (23), and parameters of blood glucose homeostasis in women without GDM. The MEHP/MECPP ratio was positively associated with 120-min plasma glucose (adjusted $\beta 0.280$, $p=0.00008$ ), explaining $7.6 \%$ of the variation in 120 -min glucose, but not any other indices of glucose homeostasis.

We hypothesised that the lack of association of MEHP and MCiOP with GDM, despite their positive associations with 120-min plasma glucose, may reflect the relatively small proportion of cases in our sample with abnormal 120-min glucose levels, owing to the use of a higher diagnostic threshold $(\geq 8.5 \mathrm{mmol} / \mathrm{l})$ $(31,32)$ than has featured in previous GDM guidelines (e.g., $\geq 7.8 \mathrm{mmol} / \mathrm{l}$ ) (33). We, therefore, repeated our GDM analyses for MEHP and MCiOP using the 1999 WHO criteria (33). 
TABLE 4 | Adjusted odd ratios and 95\% Cls of incident gestational diabetes mellitus (GDM) by category of concentrations of the different chemicals.

\begin{tabular}{|c|c|c|c|c|c|c|}
\hline Chemical & Quartile 1 & Quartile 2 & Quartile 3 & Quartile 4 & $\begin{array}{c}p \text {-Value for } \\
\text { heterogeneity }\end{array}$ & $\begin{array}{l}p \text {-Value for } \\
\text { linear trend }\end{array}$ \\
\hline \multicolumn{7}{|l|}{ Monoethyl phthalate } \\
\hline Incidence of GDM (\%) & $10 / 58(17.2 \%)$ & 14/58 (24.1\%) & 10/58 (17.2\%) & 13/58 (22.4\%) & 0.45 & 0.87 \\
\hline Adjusted OR $(95 \% \mathrm{Cl})^{\mathrm{a}}$ & Referent & $1.65(0.60-4.56)$ & $0.67(0.21-2.07)$ & $1.19(0.42-3.37)$ & & \\
\hline \multicolumn{7}{|l|}{ Monoisobutyl phthalate } \\
\hline Incidence of GDM (\%) & $9 / 58(15.5 \%)$ & 17/58 (29.3\%) & $6 / 58(10.3 \%)$ & 15/58 (25.9\%) & $0.001^{\star}$ & 0.41 \\
\hline Adjusted OR $(95 \% \mathrm{Cl})^{\mathrm{a}}$ & Referent & $5.69(1.56-20.73)^{\star}$ & $0.37(0.06-2.26)$ & $4.89(1.32-18.14)^{\star}$ & & \\
\hline \multicolumn{7}{|l|}{ Mono-n-butyl phthalate } \\
\hline Incidence of GDM (\%) & 13/66 (19.7\%) & $12 / 50(24.0 \%)$ & 9/58 (15.5\%) & 13/58 (22.4\%) & 0.26 & 0.81 \\
\hline Adjusted OR $(95 \% \mathrm{Cl})^{\mathrm{a}}$ & Referent & $1.17(0.40-3.41)$ & $0.43(0.13-1.44)$ & $1.42(0.52-3.88)$ & & \\
\hline \multicolumn{7}{|c|}{ Mono-(2-ethylhexyl) phthalate } \\
\hline Incidence of GDM (\%) & $17 / 84(20.2 \%)$ & 8/32 (25.0\%) & $11 / 58(19.0 \%)$ & 11/58 (19.0\%) & 0.53 & 0.77 \\
\hline Adjusted OR $(95 \% \mathrm{Cl})^{\mathrm{a}}$ & Referent & $2.14(0.72-6.35)$ & $1.14(0.42-3.09)$ & $1.03(0.38-2.79)$ & & \\
\hline \multicolumn{7}{|c|}{ Mono-(2-ethyl-5-carboxypentyl) phthalate } \\
\hline Incidence of GDM (\%) & $12 / 58(20.7 \%)$ & 11/58 (19.0\%) & $9 / 58(15.5 \%)$ & $15 / 58(25.9 \%)$ & 0.29 & 0.78 \\
\hline Adjusted OR $(95 \% \mathrm{Cl})^{\mathrm{a}}$ & Referent & $0.61(0.20-1.81)$ & $0.42(0.13-1.36)$ & $1.19(0.44-3.17)$ & & \\
\hline \multicolumn{7}{|c|}{ Mono(carboxyisooctyl) phthalate } \\
\hline Incidence of GDM (\%) & $9 / 58(15.5 \%)$ & 13/58 (22.4\%) & 11/58 (19.0\%) & $14 / 58$ (24.1\%) & 0.87 & 0.89 \\
\hline Adjusted OR $(95 \% \mathrm{Cl})^{\mathrm{a}}$ & Referent & $1.54(0.53-4.52)$ & $1.18(0.38-3.67)$ & $1.39(0.47-4.14)$ & & \\
\hline \multicolumn{7}{|l|}{ Bisphenol A } \\
\hline Incidence of GDM (\%) & 11/57 (19.3\%) & 15/57 (26.3\%) & 13/57 (22.8\%) & 7/57 (12.3\%) & 0.07 & 0.24 \\
\hline Adjusted OR $(95 \% \mathrm{Cl})^{\mathrm{a}}$ & Referent & $2.58(0.84-7.94)$ & $1.04(0.31-3.53)$ & $0.56(0.14-2.28)$ & & \\
\hline \multicolumn{7}{|l|}{ Triclosan } \\
\hline Incidence of GDM (\%) & $19 / 70(27.1 \%)$ & 8/44 (18.2\%) & $8 / 57$ (14.0\%) & 11/57 (19.3\%) & $0.009^{\star}$ & $0.022^{*}$ \\
\hline Adjusted OR $(95 \% \mathrm{Cl})^{\mathrm{a}}$ & Referent & $0.25(0.07-0.86)^{*}$ & $0.12(0.03-0.55)^{\star}$ & $0.35(0.12-0.98)^{\star}$ & & \\
\hline \multicolumn{7}{|l|}{ Benzophenone-3 } \\
\hline Incidence of GDM (\%) & $10 / 68(14.7 \%)$ & 9/46 (19.6\%) & 16/57 (28.1\%) & 11/57 (19.3\%) & 0.83 & 0.95 \\
\hline Adjusted OR $(95 \% \mathrm{Cl})^{\mathrm{a}}$ & Referent & $1.09(0.38-3.14)$ & $1.40(0.52-3.77)$ & $0.86(0.29-2.51)$ & & \\
\hline
\end{tabular}

${ }^{a}$ Adjusted for age, pre-pregnancy body mass index (log-transformed), IMD (log-transformed), and parity; $n=197$ for phthalates, $n=193$ for phenols. ${ }^{*} p<0.05$

In continuous models, neither MEHP (aOR per log increase in concentration $1.57,95 \%$ CI $0.88-2.78, p=0.12$ ) nor MCiOP (aOR per log increase in concentration 3.02 , 95\% CI 0.81-11.26, $p=0.10$ ) was associated with incident GDM; and MCiOP was not associated with GDM in the categorical analysis $\left(p_{\text {het }}=0.87\right.$, $\left.p_{\text {trend }}=0.46\right)$. However, women with MEHP levels in the second (aOR 25.82, 95\% CI 1.79-372.52, $p=0.017$ ) and fourth (aOR $23.22,95 \%$ CI $1.68-320.03, p=0.019$ ) quartiles, but not in the third quartile (aOR 8.09, 95\% CI 0.59-111.44, $p=0.12$ ), had significantly increased odds of GDM ( $\left.p_{\text {het }}=0.08, p_{\text {trend }}=0.041\right)$.

Of the 232 male infants born to the women in our study, 16 (6.9\%) had cryptorchidism at birth (Table 2). An additional 16 (6.9\%) infant had cryptorchidism at 3, 12, or 24 months of age, but not at birth. Congenital cryptorchidism was not associated with maternal GDM (aOR 1.46, 95\% CI 0.39-5.50, $p=0.58$ ) or fasting/stimulated glucose levels, HOMA2-B, HOMA2-IR, or disposition index (data not shown). When we remodelled the of odds of GDM in relation to serum phthalate/phenol concentrations in the 216 mothers whose sons did not have cryptorchidism at birth, our results were not substantially different from the primary analyses: triclosan was associated with incident GDM in both the continuous analysis (aOR per log increase in concentration $0.48,95 \%$ CI $0.28-0.82, p=0.007$ ) and categorical analysis $\left(p_{\text {het }}=0.027, p_{\text {trend }}=0.019\right)$, and women with MiBP levels in the second (aOR 7.33, 95\% CI 1.71-31.34, $p=0.007$ ) and fourth (aOR 5.31, 95\% CI 1.25-22.53, $p=0.024$ ) quartiles, but not in the third quartile, had higher odds of GDM ( $p_{\text {het }}=0.002$, $\left.p_{\text {trend }}=0.39\right)$ (other data not shown).

\section{DISCUSSION}

Using a prospective cohort design, we examined the associations between serum levels of phthalate metabolites, BPA, triclosan, and BP-3 measured at 10-17 weeks of gestation with GDM and indices of blood glucose homeostasis at 28 weeks of gestation. We observed a moderate inverse association between triclosan levels and GDM, and an apparent non-linear association between MiBP and GDM. We also demonstrated that MEHP, MCiOP, and the MEHP/MECPP ratio were positively associated with 120-min glucose levels but no other markers of glucose homeostasis in women without GDM. Sensitivity analyses using the 1999 WHO criteria for GDM suggested an additional nonlinear association between MEHP levels and GDM. We did not find any significant associations for BPA.

Triclosan was inversely associated with incident GDM, with no evidence of a threshold or non-linear relationship. This unexpected association persisted in a sensitivity analysis using alternative GDM diagnostic criteria and a further analysis in 
which mothers whose sons had congenital cryptorchidism were excluded. There were no concomitant associations between triclosan and markers of glucose homeostasis in non-diabetic women. The only relevant experimental study of triclosan to date showed that administration to pregnant mice at $8 \mathrm{mg} / \mathrm{kg} /$ day-several thousand times the average human intake (36) resulted in IGT with increased insulin resistance and reduced $\beta$-cell function at gestational day 17 , although this effect was not seen at lower doses (1-4 mg/kg/day) (37). An epidemiological study found no associations between urinary triclosan levels and patient-reported diabetes, fasting glucose, or fasting insulin in 1,455 non-pregnant adults (20). However, a reduction in GDM incidence could, at least in part, explain a reported inverse association between maternal triclosan levels and fetal growth in the third trimester (38). Triclosan is a broad-spectrum antimicrobial agent commonly used in personal care products such as soaps, toothpastes, and deodorants (although in 2016, it was banned from hand/body washes by the US Food and Drug Administration and the European Commission) (39). The mechanism for its apparent protective effect against GDM is unknown, but possibilities might include a reduction in systemic inflammation, and consequently insulin resistance, through its antimicrobial and/or anti-inflammatory actions $(40,41)$, or an increase in catabolism and weight loss through mitochondrial uncoupling (41). Alternatively, the strong inverse association observed between triclosan and GDM risk may reflect residual confounding due to unmeasured maternal factors: for example, triclosan levels may be indicative of health-related behaviours such as better personal care and greater health consciousness, which may in turn be protective against GDM (2).

Of the phthalate metabolites, only MiBP was associated with incident GDM in our primary analyses, although in a non-linear manner, with increased odds in the second and top quartiles only. Non-monotonic dose-response relationships are well described in the EDC literature, including for certain phthalates (42); however, the majority of such relationships are biphasic. We could not find any historical data to support a possible mechanistic explanation for the $M$-shaped relationship observed for MiBP. Furthermore, MiBP was not associated with any other markers of glucose homeostasis in our study. We, therefore, conclude that our data do not support a true effect of MiBP on GDM.

Mono-(2-ethylhexyl) phthalate and MCiOP were positively associated with 120-min plasma glucose. These findings are consistent with the in vitro actions of phthalates (4) and rodent studies of DEHP (6). Two epidemiological studies of summed DEHP metabolites (including MEHP) have demonstrated associations with T2DM $(7,9)$-although other studies assessing MEHP in isolation have not found associations with T2DM or 120 -min glucose $(7,8)$, and none have assessed glycaemia in relation to MCiOP. We also found that the MEHP/MECPP ratio was positively associated with 120 -min glucose, consistent with MEHP having greater endocrine disrupting activity than its secondary metabolites (35). The lack of association of these phthalate metabolites with fasting glucose, HOMA2-B, and HOMA2-IR could be due to a greater effect on stimulated, rather than baseline, insulin secretion, although we did not detect an association with 60-min insulin secretion (disposition index), either. We hypothesised that the lack of association with GDM in our primary analysis reflected the relatively small number of cases with abnormal 120-min glucose levels, and in a sensitivity analysis using a lower diagnostic threshold $(\geq 7.8 \mathrm{mmol} / \mathrm{l}$ rather than $\geq 8.5 \mathrm{mmol} / \mathrm{l}$ ), we found some evidence for an association between MEHP and incident GDM (significantly increased odds of GDM in the second and top quartiles only). It is possible that this association might be stronger in a population with higher MEHP levels, resulting in a greater incidence of abnormal 120min glucose levels. In support of this, an association between summed DEHP metabolites and incident T2DM was reported in a cohort of women with high urinary concentrations (median 302-325 nmol/l) but not in a cohort with lower concentrations (median 230-278 nmol/l) (9).

Our phthalate results do not corroborate with three other recent prospective studies of GDM, which variously reported inverse associations of $\mathrm{MiBP}$ and $\mathrm{MBzP}$ with 60 -min glucose (10), a positive association of MEP with IGT but an inverse association of DEHP metabolites with IGT (11), and no associations of any phthalates with IGT/GDM (12). Furthermore, we did not find any associations between BPA and incident GDM or markers of glucose homeostasis, in contrast to various epidemiological studies of BPA and T2DM $(9,15,17)$, and one previous study (18), but not two others $(12,19)$, of BPA and gestational glycaemia. These discrepancies may reflect differences in methodology, such as the matrix of measurement (urine vs. serum) (43), glucose load used (50 vs. 75 g), or gestation at phthalate/BPA measurement (first vs. second trimester) (18); or differences between the study populations and our own: for example, the three cohorts in which relationships between phthalate levels and GDM were previously assessed had higher prevalences of overweight/obesity ( $>50,45.4$, and 37.3 vs. $33.8 \%)$ and non-white ethnicity $(47.2,41.1$, and 15.5 vs. $1.7 \%)$ than our own; and our cohort did not include mothers of female infants.

The prevalence of GDM in our subcohort (20.3\%) was severalfold higher than the reported prevalence for England and Wales $(\sim 3.5 \%)$ (1). This may reflect our use of the new international criteria for GDM $(31,32)$, which differ from those used by most previous population studies (2), and from those currently advocated in England and Wales (1); indeed, using older criteria (33), the prevalence in our subcohort was markedly lower (11.6\%), although still higher than reported by most previous European population studies (0.6-22.3\%) (2). Additionally, the women in our study may not be truly representative of the entire CBGS cohort. For example, several studies have suggested that women carrying a male fetus have an increased risk of GDM (44-46), and some authors have reported an association between GDM and congenital cryptorchidism (34), although we and others have found no such link (47).

We did not have data for all possible confounders: for example, education, income, activity levels, diet, subfertility, family history of diabetes, and other EDCs $(12,18)$. This may have led to overestimation of effect sizes, as may have any antagonistic interactions between EDCs. Conversely, we cannot rule out over-adjustment in our analyses: for example, if an EDC had independent effects 
on both adiposity and glucose intolerance, then, correction for BMI would have led to an underestimation of its effect on GDM.

Serum phthalate metabolite levels in our study were of a similar order of magnitude to those reported in other cohorts of pregnant or postpartum women $(48,49)$. Serum triclosan levels were $\sim 10$-fold to 20 -fold lower than reported in Australian women of childbearing age (50), but of a similar range to those in US adults and children $(51,52)$.

Strengths of our study include its prospective design; use of laboratory criteria to diagnose GDM; sample collection and storage in glass tubes (to avoid phthalate/BPA contamination); use of a highly sensitive technique for measuring the chemicals; and our ability to account for a number of potential confounding variables. This is also the first study to examine the association between phthalate metabolites and phenols and markers of insulin resistance and $\beta$-cell function in pregnant women.

Our study has several limitations. First, the sample size was relatively small, which will have limited precision and statistical power, although it was larger than two of the five previously published studies of phthalates/BPA and GDM $(10,19)$. Second, our use of the 60-min disposition index to assess insulin secretion may have weakened associations between chemical levels and first-phase insulin secretion because the 60 -min insulinogenic index is not as robust a measure of $\beta$-cell function as the 30 -min insulinogenic index (53). Third, measurement of serum levels of the chemicals may have resulted in exposure misclassification: phthalates (24), BPA (54), and triclosan (55) all have short biological half-lives $(<24 \mathrm{~h})$, meaning that serum measurements may reflect recent brief exposures rather than the subjects' daily habits; and the levels of these chemicals can be several orders of magnitude lower in serum than urine $(24,43)$, increasing the possibility that external/iatrogenic contamination has obscured true exposures. However, we believe that our positive findings are valid, for several reasons: we took steps to avoid external contamination of serum; MCiOP and MECPP are downstream phthalate metabolites, which are not known to form as a result of external contamination (43); and it is most likely that exposure misclassification would be non-differential, which would attenuate, rather than enhance, any relationships found. The final study limitation was that we examined effects of the phthalate metabolites and phenols individually, with no allowance for mixture effects or corrections for multiple testing. However, we feel that the latter is justified by the previously demonstrated associations with glucose metabolism for many of the chemicals $(7-11,15-17)$; and indeed most significant $p$ values were $\leq 0.01$, making a type- 1 error unlikely.

In conclusion, our study provides a novel and interesting demonstration of inverse association between maternal serum triclosan levels at 10-17 weeks of gestation and incident GDM, in addition to biologically plausible associations between MEHP, MCiOP, and the MEHP/MECPP ratio and stimulated blood glucose levels. However, these findings need to be confirmed by further carefully designed prospective studies in other settings, that use a variety of specimen types (including urine) and assess exposure levels at multiple time points. More evidence is required before specific recommendations can be made with regards to clinical practice in diabetes or public health policy.

\section{ETHICS STATEMENT}

This study was carried out in accordance with the recommendations of Cambridge Local Research Ethics Committee with written informed consent from all subjects. All subjects gave written informed consent in accordance with the Declaration of Helsinki. The protocol was approved by Cambridge Local Research Ethics Committee.

\section{AUTHOR CONTRIBUTIONS}

$\mathrm{BF}, \mathrm{AT}$, and CA contributed to the conception and design of the study; BF, HF, A-MA, AJ, and AT performed the data analysis; $\mathrm{KO}$ provided statistical expertise; BF wrote the first draft of the manuscript; AT, IH, DD, KO, and CA contributed to the design of the CBGS; and HF, A-MA, AJ, IH, DD, KO, and CA contributed to data collection. All authors contributed to the interpretation of the results and revision of the manuscript for important intellectual content, and approved the final version of the manuscript.

\section{ACKNOWLEDGMENTS}

We thank the CBGS research nurses Suzanne Smith, AnneMarie Wardell, Karen Forbes, Petra Tucker, and Lesley Dark; all the families who contributed to the study; the staff at the Addenbrooke's NIHR-Wellcome Trust Clinical Research Facility and the NIHR Cambridge Comprehensive Biomedical Research Centre; and the midwives at the Rosie Maternity Hospital, Cambridge, UK.

\section{FUNDING}

This work was supported by a European Union Framework V programme (QLK4-1999-01422), the World Cancer Research Fund International (2004/03), the Medical Research Council (UK) (G1001995, U106179472), the Newlife Foundation for Disabled Children (07/20), the Evelyn Trust (EW9035322), the Mothercare Charitable Foundation (RG54608), Mead Johnson Nutrition, the National Institute for Health Research Cambridge Comprehensive Biomedical Research Centre, and the International Center for Research and Research Training in Endocrine Disruption of Male Reproduction and Child Health (EDMaRC).

\section{SUPPLEMENTARY MATERIAL}

The Supplementary Material for this article can be found online at http://www.frontiersin.org/articles/10.3389/fendo.2018.00099/ full\#supplementary-material. 


\section{REFERENCES}

1. National Collaborating Centre for Women's and Children's Health. Diabetes in Pregnancy. (2015). Available from: http://www.nice.org.uk/guidance/ng3/ evidence/full-guideline-3784285 (accessed December 4, 2016).

2. Galtier F. Definition, epidemiology, risk factors. Diabetes Metab (2010) 36:628-51. doi:10.1016/j.diabet.2010.11.014

3. Frederiksen H, Jensen TK, Jørgensen N, Kyhl HB, Husby S, Skakkebaek NE, et al. Human urinary excretion of non-persistent environmental chemicals: an overview of Danish data collected between 2006 and 2012. Reproduction (2014) 147:555-65. doi:10.1530/REP-13-0522

4. Thayer KA, Heindel JJ, Bucher JR, Gallo MA. Role of environmental chemicals in diabetes and obesity: a national toxicology program workshop review. Environ Health Perspect (2012) 120:779-89. doi:10.1289/ehp.1104597

5. Rajesh P, Balasubramanian K. Di(2-ethylhexyl)phthalate exposure impairs insulin receptor and glucose transporter 4 gene expression in L6 myotubes. Hum Exp Toxicol (2014) 33:685-700. doi:10.1177/0960327113506238

6. Gayathri NS, Dhanya CR, Indu AR, Kurup PA. Changes in some hormones by low doses of di (2-ethyl hexyl) phthalate (DEHP), a commonly used plasticizer in PVC blood storage bags \& medical tubing. Indian J Med Res (2004) 119:139-44.

7. Kuo C-C, Moon K, Thayer KA, Navas-Acien A. Environmental chemicals and type 2 diabetes: an updated systematic review of the epidemiologic evidence. Curr Diab Rep (2013) 13:831-49. doi:10.1007/s11892-013-0432-6

8. Dirinck E, Dirtu AC, Geens T, Covaci A, Van Gaal L, Jorens PG. Urinary phthalate metabolites are associated with insulin resistance in obese subjects. Environ Res (2015) 137:419-23. doi:10.1016/j.envres.2015.01.010

9. Sun Q, Cornelis MC, Townsend MK, Tobias DK, Heather Eliassen A, Franke AA, et al. Association of urinary concentrations of bisphenol A and phthalate metabolites with risk of type 2 diabetes: a prospective investigation in the nurses' health study (NHS) and NHSII cohorts. Environ Health Perspect (2014) 122:616-23. doi:10.1289/ehp.1307201

10. Robledo CA, Peck JD, Stoner J, Calafat AM, Carabin H, Cowan L, et al. Urinary phthalate metabolite concentrations and blood glucose levels during pregnancy. Int JHyg Environ Health (2015) 218:324-30. doi:10.1016/j. ijheh.2015.01.005

11. James-Todd TM, Meeker JD, Huang T, Hauser R, Ferguson KK, RichEdwards JW, et al. Pregnancy urinary phthalate metabolite concentrations and gestational diabetes risk factors. Environ Int (2016) 96:118-26. doi:10.1016/j.envint.2016.09.009

12. Shapiro GD, Dodds L, Arbuckle TE, Ashley-Martin J, Fraser W, Fisher M, et al. Exposure to phthalates, bisphenol A and metals in pregnancy and the association with impaired glucose tolerance and gestational diabetes mellitus: the MIREC study. Environ Int (2015) 83:63-71. doi:10.1016/j.envint.2015.05.016

13. Calafat AM, Ye X, Wong LY, Reidy JA, Needham LL. Exposure of the U.S. population to bisphenol A and 4-tertiary-octylphenol: 2003-2004. Environ Health Perspect (2008) 116:39-44. doi:10.1289/ehp.10753

14. Batista TM, Alonso-Magdalena P, Vieira E, Amaral MEC, Cederroth CR, Nef S, et al. Short-term treatment with bisphenol-A leads to metabolic abnormalities in adult male mice. PLoS One (2012) 7:e33814. doi:10.1371/journal. pone. 0033814

15. Rochester JR. Bisphenol A and human health: a review of the literature. Reprod Toxicol (2013) 42:132-55. doi:10.1016/j.reprotox.2013.08.008

16. Beydoun HA, Khanal S, Zonderman AB, Beydoun MA. Sex differences in the association of urinary bisphenol-A concentration with selected indices of glucose homeostasis among U.S. adults. Ann Epidemiol (2014) 24:90-7. doi:10.1016/j.annepidem.2013.07.014

17. Aekplakorn W, Chailurkit L-O, Ongphiphadhanakul B. Relationship of serum bisphenol A with diabetes in the Thai population, National Health Examination Survey IV, 2009. J Diabetes (2015) 7:240-9. doi:10.1111/1753-0407.12159

18. Chiu Y-H, Mínguez-Alarcón L, Ford JB, Keller M, Seely EW, Messerlian C, et al. Trimester-specific urinary bisphenol A concentrations and blood glucose levels among pregnant women from a fertility clinic. J Clin Endocrinol Metab (2017) 102:1350-7. doi:10.1210/jc.2017-00022

19. Robledo C, Peck JD, Stoner JA, Carabin H, Cowan L, Koch HM, et al. Is bisphenol-A exposure during pregnancy associated with blood glucose levels or diagnosis of gestational diabetes? J Toxicol Environ Health A (2013) 76:865-73. doi:10.1080/15287394.2013.824395
20. Lang IA, Galloway TS, Scarlett A, Henley WE, Depledge M, Wallace RB, et al. Association of urinary bisphenol A concentration with medical disorders and laboratory abnormalities in adults. JAMA (2008) 300:1303-10. doi:10.1016/S0145-4145(09)79528-0

21. Prentice P, Acerini CL, Eleftheriou A, Hughes IA, Ong KK, Dunger DB. Cohort profile: the Cambridge Baby Growth Study (CBGS). Int J Epidemiol (2015) 45(1):35g-35g. doi:10.1093/ije/dyv318

22. Department for Communities and Local Government. The English Indices of Deprivation 2007. London: Department for Communities and Local Government (2007).

23. Joensen UN, Frederiksen H, Blomberg Jensen M, Lauritsen MP, Olesen IA, Lassen TH, et al. Phthalate excretion pattern and testicular function: a study of 881 healthy Danish men. Environ Health Perspect (2012) 120:1397-403. doi:10.1289/ehp.1205113

24. Frederiksen H, Jørgensen N, Andersson A-M. Correlations between phthalate metabolites in urine, serum, and seminal plasma from young Danish men determined by isotope dilution liquid chromatography tandem mass spectrometry. J Anal Toxicol (2010) 34:400-10. doi:10.1038/jes.2010.6

25. Frederiksen H, Aksglaede L, Sorensen K, Nielsen O, Main KM, Skakkebaek NE, et al. Bisphenol A and other phenols in urine from Danish children and adolescents analyzed by isotope diluted TurboFlow-LC-MS/MS. Int J Hyg Environ Health (2013) 216:710-20. doi:10.1016/j.ijheh.2013.01.007

26. Hornung RW, Reed LD. Estimation of average concentration in the presence of nondetectable values. Appl Occup Environ Hyg (1990) 5:46-51. doi:10.1080/ 1047322X.1990.10389587

27. Sherard GB, Newton ER. Is routine hemoglobin and hematocrit testing on admission to labor and delivery needed? Obstet Gynecol (2001) 98:1038-40. doi:10.1016/S0029-7844(01)01586-1

28. Fogh-Anderson N, Wimberley PD, Thode J, Siggaard-Andersen O. Direct reading glucose electrodes detect the molality of glucose in plasma and whole blood. Clin Chim Acta (1990) 189:33-8. doi:10.1016/0009-8981(90) 90232-H

29. Wallace TM, Levy JC, Matthews DR. Use and abuse of HOMA modeling. Diabetes Care (2004) 27:1487-95. doi:10.2337/diacare.27.6.1487

30. Utzschneider KM, Prigeon RL, Faulenbach MV, Tong J, Carr DB, Boyko EJ, et al. Oral disposition index predicts the development of future diabetes above and beyond fasting and 2-h glucose levels. Diabetes Care (2009) 32:335-41. doi: $10.2337 / \mathrm{dc} 08-1478$

31. International Association of Diabetes and Pregnancy Study Groups Consensus Panel, Metzger BE, Gabbe SG, Persson B, Buchanan TA, Catalano PA, et al. International association of diabetes and pregnancy study groups recommendations on the diagnosis and classification of hyperglycemia in pregnancy. Diabetes Care (2010) 33:676-82. doi:10.2337/dc09-1848

32. World Health Organization. Diagnostic Criteria and Classification of Hyperglycaemia First Detected in Pregnancy. (2013). Available from: http:// www.who.int/diabetes/publications/Hyperglycaemia_In_Pregnancy/en/ (accessed December 4, 2016).

33. Alberti KG, Zimmet PZ. Definition, diagnosis and classification of diabetes mellitus and its complications. Part 1: diagnosis and classification of diabetes mellitus provisional report of a WHO consultation. Diabet Med (1998) 15:539-53. doi:10.1002/(SICI)1096-9136(199807)15:7<539:: AID-DIA668>3.0.CO;2-S

34. VirtanenHE,TapanainenAE,KalevaMM,SuomiAM,MainKM,SkakkebaekNE, et al. Mild gestational diabetes as a risk factor for congenital cryptorchidism. J Clin Endocrinol Metab (2006) 91:4862-5. doi:10.1210/jc.2006-1420

35. Chauvigné F, Menuet A, Lesné L, Chagnon M-C, Chevrier C, Regnier J-F, et al. Time- and dose-related effects of di-(2-ethylhexyl) phthalate and its main metabolites on the function of the rat fetal testis in vitro. Environ Health Perspect (2009) 117:515-21. doi:10.1289/ehp.11870

36. Calafat AM, Ye X, Wong L-Y, Reidy JA, Needham LL. Urinary concentrations of triclosan in the U.S. population: 2003-2004. Environ Health Perspect (2008) 116:303-7. doi:10.1289/ehp.10768

37. Hua X, Cao X-Y, Wang X-L, Sun P, Chen L. Exposure of pregnant mice to triclosan causes insulin resistance via thyroxine reduction. Toxicol Sci (2017) 36(6):777-89. doi:10.1093/toxsci/kfx166

38. Philippat C, Botton J, Calafat AM, Ye X, Charles M-A, Slama R. Prenatal exposure to phenols and growth in boys. Epidemiology (2014) 25:625-35. doi:10.1097/EDE.0000000000000132 
39. McNamara PJ, Levy SB. Triclosan: an instructive tale. Antimicrob Agents Chemother (2016) 60:7015-6. doi:10.1128/AAC.02105-16

40. Cullinan MP, Westerman B, Hamlet SM, Palmer JE, Faddy MJ, Seymour GJ. The effect of a triclosan-containing dentifrice on the progression of periodontal disease in an adult population. JClin Periodontol (2003) 30:414-9. doi:10.1034/j.1600-051X.2003.20030.x

41. Weatherly LM, Shim J, Hashmi HN, Kennedy RH, Hess ST, Gosse JA. Antimicrobial agent triclosan is a proton ionophore uncoupler of mitochondria in living rat and human mast cells and in primary human keratinocytes. J Appl Toxicol (2015) 36(6):777-89. doi:10.1002/jat.3209

42. Lagarde F, Beausoleil C, Belcher SM, Belzunces LP, Emond C, Guerbet M, et al. Non-monotonic dose-response relationships and endocrine disruptors: a qualitative method of assessment. Environ Health (2015) 14:13. doi:10.1186/1476-069X-14-13

43. Calafat AM, Koch HM, Swan SH, Hauser R, Goldman LR, Lanphear BP, et al. Misuse of blood serum to assess exposure to bisphenol A and phthalates. Breast Cancer Res (2013) 15:403. doi:10.1186/bcr3494

44. Retnakaran R, Kramer CK, Ye C, Kew S, Hanley AJ, Connelly PW, et al. Fetal sex and maternal risk of gestational diabetes mellitus: the impact of having a boy. Diabetes Care (2015) 38:844-51. doi:10.2337/dc14-2551

45. Retnakaran R, Shah BR. Fetal sex and the natural history of maternal risk of diabetes during and after pregnancy. JClin Endocrinol Metab (2015) 100:2574-80. doi:10.1210/jc.2015-1763

46. Sheiner E, Levy A, Katz M, Hershkovitz R, Leron E, Mazor M. Gender does matter in perinatal medicine. Fetal Diagn Ther (2004) 19:366-9. doi:10.1159/000077967

47. Trabert B, Chodick G, Shalev V, Sella T, Longnecker MP, McGlynn KA. Gestational diabetes and the risk of cryptorchidism and hypospadias. Epidemiology (2014) 25:152-3. doi:10.1097/EDE.0000000000000014

48. Hart R, Doherty DA, Frederiksen H, Keelan JA, Hickey M, Sloboda D, et al. The influence of antenatal exposure to phthalates on subsequent female reproductive development in adolescence: a pilot study. Reproduction (2014) 147:379-90. doi:10.1530/REP-13-0331

49. Hines EP, Calafat AM, Silva MJ, Mendola P, Fenton SE. Concentrations of Phthalate metabolites in milk, urine, saliva, and serum of lactating North
Carolina women. Environ Health Perspect (2009) 117:86-92. doi:10.1289/ ehp. 11610

50. Allmyr M, Harden F, Toms LML, Mueller JF, McLachlan MS, AdolfssonErici $\mathrm{M}$, et al. The influence of age and gender on triclosan concentrations in Australian human blood serum. Sci Total Environ (2008) 393:162-7. doi:10.1016/j.scitotenv.2007.12.006

51. Ye X, Tao LJ, Needham LL, Calafat AM. Automated on-line column-switching HPLC-MS/MS method for measuring environmental phenols and parabens in serum. Talanta (2008) 76:865-71. doi:10.1016/j.talanta.2008.04.034

52. Ye X, Zhou X, Wong LY, Calafat AM. Concentrations of bisphenol a and seven other phenols in pooled sera from 3-11 year old children: 2001-2002 national health and nutrition examination survey. Environ Sci Technol (2012) 46:12664-71. doi:10.1021/es303109c

53. Tura A, Kautzky-Willer A, Pacini G. Insulinogenic indices from insulin and C-peptide: comparison of beta-cell function from OGTT and IVGTT. Diabetes Res Clin Pract (2006) 72:298-301. doi:10.1016/j.diabres.2005.10.005

54. Vandenberg LN, Hauser R, Marcus M, Olea N, Welshons WV. Human exposure to bisphenol A (BPA). Reprod Toxicol (2007) 24:139-77. doi:10.1016/j. reprotox.2007.07.010

55. Sandborgh-Englund G, Adolfsson-Erici M, Odham G, Ekstrand J. Pharmacokinetics of triclosan following oral ingestion in humans. J Toxicol Environ Health A (2006) 69(20):1861-73. doi:10.1080/15287390600631706

Conflict of Interest Statement: The authors declare that the research was conducted in the absence of any commercial or financial relationships that could be construed as a potential conflict of interest.

Copyright $\odot 2018$ Fisher, Frederiksen, Andersson, Juul, Thankamony, Ong, Dunger, Hughes and Acerini. This is an open-access article distributed under the terms of the Creative Commons Attribution License (CC BY). The use, distribution or reproduction in other forums is permitted, provided the original author(s) and the copyright owner are credited and that the original publication in this journal is cited, in accordance with accepted academic practice. No use, distribution or reproduction is permitted which does not comply with these terms. 